\title{
Barriers and Strategies of Overcoming Barriers in Healthy Nutritional Behaviors from Women's Perspective
}

\section{ART ICLE INF O}

\section{Article Type}

Qualitative Study

\section{Authors}

Abdi N. ${ }^{1}$ PhD,

Zamani-Alavijeh $\mathrm{F}^{2} P h D$

Taghdisi M.H. ${ }^{3} P h D$,

Sadeghi R. ${ }^{* 4} P h D$,

Shojaeezadeh D. ${ }^{4} P h D$,

Shahsavari S. ${ }^{5} M A$

Zarezadeh $\mathrm{Y}^{1} \mathrm{PhD}$

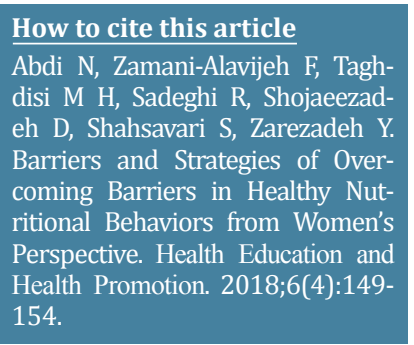

${ }^{1}$ Social Determinants of Health Research Center, Kurdistan University of Medical Sciences, Sanandaj, Iran ${ }^{2}$ Health Education \& Promotion Department, Health Faculty, Isfahan University of Medical Sciences, Isfahan, Iran

${ }^{3}$ Health Promotion \& Education Department, Health Faculty, Iran University of Medical Sciences, Tehran, Iran

${ }^{4}$ Health Promotion \& Education Department, Health Faculty, Tehran University of Medical Sciences, Tehran, Iran

${ }^{5}$ Public Health Department, Health Faculty, Kurdistan University of Medical Sciences, Sanandaj, Iran

\section{*Correspondence}

Address: Health Promotion \& Education Department, Health Faculty, Tehran University of Medical Sciences, Poursina Street, Qods Street, Enghelab Street, Tehran, Iran. Postal Code: 1417613151

Phone: +98 (21) 88955888

Fax: +98 (21) 88989129

sadeghi_roya@yahoo.co.uk

\section{Article History}

Received: August 19, 2017

Accepted: June 02, 2018

ePublished: November 30, 2018

\section{A B S T R A C T}

Aims Health and behavior are closely related subjects because lots of diseases are rooted in individuals' unhealthy behaviors and habits. The current study aimed at identifying barriers and strategies of overcoming barriers in healthy nutritional behaviors in women.

Participants \& Methods This qualitative study was conducted in 2014 based on content analysis. The participants were 50 married women with the age range of 18 to 50 years old referring to 4 healthcare centers in Sanandaj, who were selected by purposive sampling. Using semi-structured interviews, the data were collected through group discussions and individual in-depth interviews. A thematic analysis approach was applied for data analyses and MAXQDA 10 software was employed to analyze the data.

Findings Of the total interviews and discussion groups, 200 initial codes were obtained and they were grouped into 4 categories, including individual barriers, social barriers, overcoming individual barriers, and overcoming social barriers. Lack of awareness and healthy cooking skills, unhealthy diet of parents as a negative role model, laziness of wives and women, lack of time, lack of mental relaxation, illiteracy economic issues, and the role of government were mentioned by the participants as individual and social barriers.

Conclusion Barriers in healthy nutritional behaviors from women's perspective are devided into individual and social barriers and some strategies are mentioned to overcome these barriers, including learning required skills in terms of healthy diet/nutrition, raising awareness, time management, monitoring the contaminated foods by the government, providing public information, training through media, and resolving economic problems.

Keywords Health Behavior; Qualitative Research; Women

\section{CIT A T I O N L I N KS}

[1] Health services ... [2] Exploring health promoting lifestyle behaviors of Japanese college women: Perceptions, practices ... [3] Assessing the micronutrient and macronutrient intakes in female students and comparing them with the set ... [4] Barriers of healthy nutrition from the perspective men in Tehran: A qualitative... [5] Application of qualitative methods in program planning for health promotion ... [6] Youth understanding of healthy eating and obesity: A focus group ... [7] Formative assessment using social marketing principles to identify health and nutrition perspectives of Native American women living within the Chickasaw Nation boundaries in ... [8] A qualitative study exploring socio-economic differences in parental lay knowledge of food and health: Implications for public health ... [9] I eat healthfully but I am not a freak, Consumers' everyday life perspective on healthful ... [10] Using interpretative phenomenology to understand the food-related experiences and beliefs of a select group of low-income UK ... [11] A qualitative study exploring how mothers manage their teenage children's ... [12] Implications of qualitative research for nutrition education geared to selected Hispanic ... [13] Attitudes to lifestyle risk factors for coronary heart disease amongst South Asians in Leicester: A focus group ... [14] The development of a feasible communityspecific cardiovascular disease prevention program: Triangulation of methods and ... [15] Factors related to cardiovascular disease risk reduction in midlife and older women: A qualitative ... [16] Three approaches to qualitative content ... [17] Progress in organizational justice: Tunneling through the ... [18] In search of how people change. Applications to addictive ... [19] Stage theories of health behavior: conceptual and methodological ... [20] Effective factors on nutritional behavior of female workers based on integrated model of planned behavior and self-efficacy: A qualitative ... [21] Nutrition knowledge and health food ... [22] Behavioral determinants of cardiovascular diseases risk factors: A qualitative directed content ... [23] Identification of barriers and facilitators of healthy food choices among children aged 6 to 12 years: From the Kahnawake schools diabetes prevention ... [24] Qualitative developmental research among low income African American adults to inform a social marketing campaign ... 
Barriers and Strategies of Overcoming Barriers in Healthy...

\section{Introduction}

One of the most influential factors in the incidence of the diseases is improper lifestyle and its transformation. Generally, 53\% of causes of death is associated with individuals' lifestyles [1]. Positive behavior patterns such as proper diet, regular exercise and physical activities, and avoidance of alcohol and tobacco consumption play a significant role in individual health promotion [2].

Lifestyle modification requires changing behaviors, which constitute a major part of daily life habits of individuals [2]. Healthy diet and consumer culture are considered as the most important dimensions of lifestyle and the most effctive one in promoting public health. The main goal of these two factors are to provide appropriate physical-mental condition in terms of growth and development of organisms as well as mobility and efficiency of human beings in their social lives.

Generally, good nutrition and healthy eating lead to general and stable health of society [3]. Food habits and culture are interrelated, i.e. nutritional behavior patterns of a society is a part of their cultural patterns. In other words, beliefs, values, and social norms have a role in forming nutritional habits [4].

Being aware of opinions, demands, needs, preferences, behaviors, and beliefs of customers is regarded as a basic and important principle and plays a vital role in designing intervention programs to promote healthy eating behaviors. Therfore, relying on conditions and real needs of people and considering their beliefs and sanitary norms, studies that are able to present programs to modify the nutritional pattern of society are of top health priorities of today [4].

Qualitative methods that collect in-depth data can assist health trainers, so that they can find the origin of health-related problems and behavioral and environmental factors from the viewpoint of those who are directly involved with problems. These trainers can also achieve a comprehensive approach in this regard [5]. Qualitative approaches are capapble of familiarizing us with latent layers of behavior. Since nutritional behavior is a series of beliefs and habits relying on social norms and values, we need to deeply discover the behavior in its cultural context before making any plan to improve it.

Different qualitative studies have been conducted in terms of examining and understanding views [6-9], beliefs and experiences [10], mothers management in nutritional behaviors [11], and nutrition training [12] as well as barriers to healthy eating behaviors [13-15]. Obviously, forming nutritional beliefs is considered as a sub-culture, which, like other social phenomena, is of great importance.

Therfore, regarding the healthy diet barriers, conducting exploratory studies and taking sociocultural aspects of forming healthy diet barriers process into consideration seems essential. These issues help health practitioners consider different possibilities in planning and developing any training interventions.

According to the variety of eating behaviors in various cultures, the anonymity of barriers to eating behaviors, and their consequences, the present research was conducted with the aim of examining the barriers of healthy eating behaviors and strategies to overcome them.

\section{Participants and Methods}

This qualitative study was conducted in 2014 based on content analysis. The participants were 18- to 50year-old married women referring to 4 healthcare centers in Sanandaj, who were selected by purposive sampling. They participated in the syudy freely with informed and writeen consent. The critrion for the adequacy of research sample was data saturation and, finally, 50 women covered by healthcare center participated in the study.

Using semi-structured interviews, the data were collected through group discussions and individual in-depth interviews. During 5 sessions of discussion groups and with minimum 6 and maximum 10 people in each group, and 8 individual interviews, the data were collected. The time for discussion groups and individual interviews were between 1 and 2 and, 1 and 1.5 hours, repectively. This time varied, depending on the condition and process of the sessions or interviews. The interviews began raising general questions. For example, how do you define a healthy diet? Afterwards and gradually, deeper questions, in line with the research questions, were raised. For instance, what are the barriers that stop you from having a healthy diet? Interviews began from late October 2015 and ended in late June 2016, till data saturation. In the last interview, no new data were obtained. After gathering the data, content analysis was employed [16]. Immediately, after each session, on the day of interview, all recorded interviews were transcribed thoroughly and in details. To fully understamd the situation and get involved feelingly in the process of the interviews, the interviews were listened and the transcribed data were studied by the researcher several times. Then, coding (converting the semantic and meaningful units to shorter, understandable, and desired concepts) was done. To identify similarities and differences, to merge similar codes, and to form categories, the codes were reviewed. Next, subcategories were classified based on similarities, differences, and appropriateness and categories were extracted as well. Finally, reconsidering categories, subcategories, codes, and data, main themes of the study (structures of social marketing model in this study were product, price, place, and promotion) were extracted.

To ensure the accuracy and reliability of the 


\section{1}

obtained qualitative data, criteria such as acceptability, reliability, stability, and transmissibility were considered [17]. Long-term involvement of the researcher with the research topic, data, and observing extracted codes and also immediate transcription, detailed record of all steps of the research, providing similar position for participants can all be considered as the approval of acceptability research. Some parts of interviews along with the extracted codes, categoroes, and subcategories were assessed by some observers outside the research team, familiar with qualitative research methods. Sampling in the study was done with maximum diversity, which helps with transmissibility increase of the results. MAXQDA 10 software was employed to analyze the data.

\section{Findings}

Of the total interviews and discussion groups, 200 initial codes were obtained and grouped into 4 categories during analysis process, including individual barriers, social barriers, overcoming individual barriers, and overcoming social barriers.

Individual barriers: Lack of awareness and healthy cooking skills, unhealthy diet of parents as a negative role model, laziness of wives and women, lack of time, lack of mental relaxation, and illiteracy were all mentioned by the participants. Among them, lack of aawareness was reported with increasing frequency. Take, for instance, this participant who is a good example in this case. She told us "all of us, for example, have frequentltly heard that Coca Cola is bad. Better not to eat it; or cheese puff is the cause of illness. I just thought that harmfulness of Coca Cola was due to its carbonate. See, we are not aware enough! I read in newspaper that Coca Cola is the best cleaner for cars and ceramics. As long as we know little about lots of things, we will face many problems."

Social barriers: Some subcategories of social barriers such as economic issues and inflation, fertilizers, the role of government in putting people's health in danger, and lack of raising awareness by physicians were mentined by participants. The biggest barriers mentioned by participants were inflation and economic problems. In this regard, one told "the biggest problem in our society tody is monetary problem; people are poor. Not having enough money puts our health and whole life in danger. When people can't afford to buy things or buy low-quality stuff, their health would be in danger. Why can I afford to buy meat twice a month, while another one can afford to buy everyday? How can I claim that I have a healthy diet, being in such a condition?"

Another important factor mentioned by most participants was the role of government. "From my point oif view, a participant said the government plays a significant role. People can do nothing in this regard. I really don't know who is responsible. A
Abdi N. et al.

great dissersvice is done to people's health. The government has role in unhealthy diet. The government, for example, should take care of unhealthy vegetables we consume. Why should they be contaminated?"

Overcoming individual barriers: The most important healthy nutritional behavior mentioned by the participants had to do with learning required skills in terms of healthy diet/nutrition. One of them, who believed in learning such skills, told "we don't know much about the nutrition of adults and children; even if we know something, we don't know how to implement it. We have rice in most of our meals. This is because we don't know how to cook other healthy foods without rice. We don't have ability to prepare healthy and nutritious dishes with those stuff we buy. If we develop the proper cooking skills, the diseases will decrease."

Raising awareness was another factor addressed by the majority of interviewees as an important solution to overcome existing barriers to healthy nutrition. A participant told that “Maybe I don't read about this and that is why we don't eat properly. People aren't aware enough. Doctors used to talk about different kinds of slow/fast cook pots or pressure cooker (such as Geepas, a famous brand in Iran), but these days no one talks about them. People's awareness should be raised. All are not literate. On channel 3, there is a good program, which teaches us how to cook good food and gives us useful information, but not all watch it."

Time management was mentioned by most of the participants as one of the solutions of overcoming unhealthy eating behaviors. To clarify this issue, one example was a participant's statement. She told, "I am mother of 4 kids, spending a lot of time helping them with their lessons and homework. I suffer from the lack of time for cooking and my kids have anemia and they are weak. I have to spend more time on their nutrition and it needs time management."

Overcoming social barriers: Monitoring the contaminated foods by the government, providing public information and training through media, reducing inflation, and solving economic problems were all stated by the participants. An interviewee mentioned that "society condition is the reason of all diseases; everything is artificial and contains chemicals. People can do nothing in this case. Nor fruit neither meat have nutritional values; what can a mother do? Food products should be monitored carefully. Everyday a news is broken about contaminations. We are pessimistic about everything. No one stops them." Another participant believed that "media such as TV and newspaper can play a very significant role. For example, television should show the negative effects of fast food, so that people's awareness is raised and choose healthy food except for those that don't care about their health." 
Barriers and Strategies of Overcoming Barriers in Healthy...

Also, most participants mentioned inflation and economic problems. For instance, one of them explained that "our main problem is not ignorance. It is mainly money. I know due to my kid's anemia, I should provide meat and beef liver, but is it possible with such a high inflation? As long as people have bad financial situation, the diseases will increase. The only solution is to decrease the price of food products, so that people can afford to buy them."

Interviewees were asked to suggest a mechanism to send and release messages related to healthy nutrition. Most of them agreed on holding classes in healthcare centers. Also, informing people through written materials, television, and mosques was extracted from the content of interviews.

\section{Discussion}

In order to form and implement any healthy behavior, there are some barriers both on the individual and the social levels. Likewise, healthy eating is one of the behaviors, which has its own barriers. If a person wants to make changes in his/her own eating behaviors, s/he must overcome the individual barriers; furthermore, some strategies should be designed and implemented to remove social barriers. Making sure that whatever is ultimately expressed as an intervention grants people's needs and demands, is one of the most significant duties of health staff and experts. Accordingly, identifying the barriers is the fundamental step in designing the best intervention reflecting the demands and the benefits of behavior. For everyone, in any situattion, taking an action requires a cost, at least to try to overcome our daily actions and attain what we want.

The obtained evidences from a long series of studies done by Prochaska et al. lead us to this fact that the more consumers get closer to take the action, i.e. the more they get closer to make the final decision, the more important the decision-making about the prices will be [18].

As Weinstein et al. mentined, barrires, especially short-term barriers, are by no means certain, while many benefits of social behaviors are presumptive [19]. In this study, the participants mentined barriers such as lack of awareness, lack of healthy cooking skills, unhealthy diet of parents as a negative role model, laziness of wives and women, lack of time, lack of mental relaxation, and illiteracy. Among them, lack of awarenss was mentined by participants many times, which was in line with the results of studies conducted by Keshavarz et al. and Seagert and Young [20,21]. Some subcategories of social barriers such as economic issues and inflation, fertilizers, the role of government in putting people's health in danger, and lack of raising awareness by physicians were mentined by the participants.

The biggest barrier mentioned by the participants was inflation and economic problems. These findings were in line with the findings of other studies in many aspects. The findings of a study conducted by Sabzemakan et al., for example, were consistent with the findings of this study in terms of the high cost of food, lack of easy access to healthy foods, fertilizers, lack of healthy food policies, as well as laziness and impatience [22].

In a study carried out by Parker, lack of social support and conflict between food preferences of family members were mentioned by most participants. Other barriers, from their ponits of view, were cost of food, lack of access to foods, and lack of time [9].

The result of Pierre et al.'s study were similar to Parkers's [23]. Keshavarz et al. found out that for female wokers, lack of time was the most important barrier to have a healthy diet. Other factors were fatigue and lack of motivation, lack of awareness as well as lack of acccess to healthy foods [20].

Considering the role of social and environmental factors as barriers to healthy diet, health policy makers with a comprehensive view should make a plan to improve environmental and social conditions, employing health promotion strategies along with trainings tailored to the needs of the target group as well as paying attention to cultural, social, and economic conditions of society. Given that there are some barriers in changing nutritional behaviors, there must be some strategies to overcome them. Learning healthy diet skills, raising awareness, and time management as individual strategies, and monitoring the contaminated foods by the government, providing public information, training through media, reducing inflation, and solving economic problems as social strategies were mentioned by the participants to overcome barriers to healthy diet. In Wilson's study, participants mentioned some strategies to overcome the barriers to hiking, including better street lighting and traffic control, street gangs control, and clearing stray dogs from neighborhood [24].

Through deep understanding of women's nutritional behavior barriers and overcoming barriers, the results of this qualitative study provides a solid foundation for development and designing interventions to nutritional litracy promotion based on their needs. Since most participants wanted to learn healthy diet skills, the designed interventions to healthy nutritional behavior should be based on empowering women and providing facilitator factors of healthy diet. Also, while designing, with a holistic perspective, individual and social aspects of healthy diet should be taken into account. In addition, within the framework of exchange theory, the benefits of healthy behavior should be increased and its barriers should be reduced, so that the customer reaches his/her goal, that is to promote his/her nutritional behavior and nutritional literacy. Regarding the present qualitative research, which is conducted based on social marketing model, there is no similar research. There was no limitation in the 
present study.

It is suggested that more qualitative studies be done by the application of health education models. Also, since in this study barriers and overcoming barriers strategy are found from the viewpoint of women, it is suggested that development studies be conducted to reduce nutritional barriers. In addition, presenting the results of the present study to health policy makers will be effective. Accordingly, they can plan to reduce nutritional barriers, which will lead to the promotion of health within society.

\section{Conclusion}

Barriers in healthy nutritional behaviors from women's perspective are devided into individual and social barriers and some strategies are mentioned to overcome these barriers, including learning required skills in terms of healthy diet/nutrition, raising awareness, time management, monitoring the contaminated foods by the government, providing public information, training through media, and resolving economic problems.

Acknowledgements: We would like to express our gratitude to all those who helped us to carry out this study.

Ethical Permissions: This study was approved by the Ethics Committee of Kurdistan University of Medical Sciences. (1396/21).

Conflict of Interests: There was no conflict of interest.

Authors' Contribution: Abdi N. (First author), Introduction author/Original researcher/Discussion author (16\%); Zamani-Alavijeh F. (Second author), Methodologist/Assistant (14\%); Taghdisi M.H. (Third author), Methodologist/Assistant (14\%); Sadeghi R. (Fourth author), Methodologist/Assistant/Statistical analyst (14\%); Shojaeezadeh D. (Fifth author), Assistant (14\%); Zarezadeh Y. (Sixth author), Assistant (14\%); Shahsavari S (Seventh author), Assistan.

Funding: None declared.

\section{Refrences}

1- Park J. Health services principals. Shojaee Tehrani H, translator. Tehran: Samat; 2004. p. 42. [Persian]

2- Tashiro J. Exploring health promoting lifestyle behaviors of Japanese college women: Perceptions, practices, and issues. Health Care Women Int. 2002;23(1):59-70.

3- Mirzaeian S, Ghiasvand R, Sadeghian F, Sheikhi M, Khosravi ZS, Askari G, et al. Assessing the micronutrient and macronutrient intakes in female students and comparing them with the set standard values. J Educ Health Promot. 2013;2:1.

4- Ramezani Tehrani F, Farahmand M, Amiri P, Ghanbarian A, Azizi F. Barriers of healthy nutrition from the perspective men in Tehran: A qualitative study. Payesh. 2012;11(5):725-35. [Persian]

5- Farquhar SA, Parker EA, Schulz AJ, Israel BA. Application of qualitative methods in program planning for health promotion interventions. Health Promot Pract.
2006;7(2):234-42.

6- Sylvetsky AC, Hennink M, Comeau D, Welsh JA, Hardy T, Matzigkeit $\mathrm{L}$, et al. Youth understanding of healthy eating and obesity: A focus group study. J Obes. 2013;2013:670295.

7- Parker S, Hunter T, Briley C, Miracle S, Hermann J, Van Delinder J, et al. Formative assessment using social marketing principles to identify health and nutrition perspectives of Native American women living within the Chickasaw Nation boundaries in Oklahoma. J Nutr Educ Behav. 2011;43(1):55-62.

8- Coveney J. A qualitative study exploring socio-economic differences in parental lay knowledge of food and health: Implications for public health nutrition. Public Health Nutr. 2005;8(3):290-7.

9- Bouwman LI, Te Molder H, Koelen MM, Van Woerkum CM. I eat healthfully but I am not a freak, Consumers' everyday life perspective on healthful eating. Appetite. 2009;53(3):390-8.

10- Dibsdall LA, Lambert N, Frewer LJ. Using interpretative phenomenology to understand the foodrelated experiences and beliefs of a select group of lowincome UK women. J Nutr Educ Behav. 2002;34(6):298309.

11- Chapman K, Ogden J. A qualitative study exploring how mothers manage their teenage children's diets. Vulnerable Child Youth Stud. 2009;4(1):90-100.

12- Gans KM, Joan Lovell H, Fortunet R, Mc Mahon C, Carton-Lopez S, Lasater TM. Implications of qualitative research for nutrition education geared to selected Hispanic audiences. J Nutr Educ. 1999;31(6):331-8.

13- Farooqi A, Nagra D, Edgar T, Khunti K. Attitudes to lifestyle risk factors for coronary heart disease amongst South Asians in Leicester: A focus group study. Fam Pract. 2000;17(4):293-7.

14- Nakkash R, Afifi Soweid RA, Nehlawi MT, ShediacRizkallah MC, Hajjar TA, Khogali M. The development of a feasible community-specific cardiovascular disease prevention program: Triangulation of methods and sources. Health Educ Behav. 2003;30(6):723-39.

15- Folta SC, Goldberg JP, Lichtenstein AH, Seguin R, Reed PN, Nelson ME. Factors related to cardiovascular disease risk reduction in midlife and older women: A qualitative study. Prev Chronic Dis. 2008;5(1):A06.

16- Hsieh HF, Shannon SE. Three approaches to qualitative content analysis. Qual Health Res. 2005 Nov;15(9):127788.

17- Polit DF, Beck CT. Nursing Research: Principle and Method. $6^{\text {th }}$ Edition. Philadelphia: Lippincott Williams \& Wilkins; 2004.

18- Prochaska JO, DiClemente CC, Norcross JC. In search of how people change. Applications to addictive behaviors. Am Psychol. 1992;47(9):1102-14.

19- Weinstein ND, Rothman AJ, Sutton SR. Stage theories of health behavior: conceptual and methodological issues. Health Psychol. 1998;17(3):290-9.

20- Keshavarz Z, Simbar M, Ramezankhani A. Effective factors on nutritional behavior of female workers based on integrated model of planned behavior and self-efficacy: A qualitative approach. Hakim Health Syst Res J. 2010;13(3):199-209. [Persian]

21- Seagert J, Young EA. Nutrition knowledge and health food consumption. Nutr Behav. 1983;1(2):103-13.

22- Sabzmakan L, Morowatisharifabad MA, Mohammadi E, Mazloomy-Mahmoodabad SS, Rabiei K, Naseri MH, et al. Behavioral determinants of cardiovascular diseases risk 
Barriers and Strategies of Overcoming Barriers in Healthy...

factors: A qualitative directed content analysis. ARYA Atheroscler. 2014;10(2):71-81.

23- Pierre N, Receveur O, Macaulay AC, Montour L. Identification of barriers and facilitators of healthy food choices among children aged 6 to 12 years: From the Kahnawake schools diabetes prevention project. Ecol Food
Nutr. 2007;46(2):101-23.

24- Wilson DK, St George SM, Trumpeter NN, Coulon SM, Griffin SF, Wandersman A, et al. Qualitative developmental research among low income African American adults to inform a social marketing campaign for walking. Int J Behav Nutr Phys Act. 2013;10:33. 